\title{
SIMULATION OF MULTIVARIATE SCHEDULING OPTIMIZATION FOR OPEN PRODUCTION LINE BASED ON IMPROVED GENETIC ALGORITHM
}

\author{
Li, H. Y.,**,* Gui, C. ${ }^{* *} \&$ Xiao, K.** \\ "State Key Laboratory for Information Engineering in Surveying, Mapping and Remote Sensing, \\ Wuhan University, Wuhan 430079, China \\ ** School of Information Engineering, Hubei University of Economics, Wuhan 430205, China \\ E-Mail: hongyanli2000@126.com, gui_chao@126.com,mrs_kunxiao@163.com \\ ( ${ }^{\#}$ Corresponding author)
}

\begin{abstract}
This paper attempts to overcome the defects of bottleneck recognition and scheduling optimization in open production line. For this purpose, the author analysed the impact mechanism of external disturbances and system configuration changes on the production line, and put forward a multibottleneck identification model for production line through computer simulation. Then, the proposed model was applied to optimize the scheduling of open production line. Specifically, the bottlenecks of production line were identified based on hierarchical clustering and multi-attribute decision-making, aiming to overcome the small candidate set and low accuracy of traditional bottleneck identification algorithms. The measured results show that the proposed algorithm has clear primary and secondary logics; the number of main bottleneck clusters decreased with the increase in the order; the number of machines in the main bottleneck cluster changed nonlinearly. The traditional genetic algorithm (GA) was improved in three aspects: the local optimum trap was avoided by enhancing population diversity; the iteration speed was accelerated with the introduction of adaptive crossover operator and genetic operator; without sacrificing the computing speed, the convergence quality was guaranteed through the addition of multivariate competition algorithm. The research findings provide new insights into the efficient operation of production line.

(Received, processed and accepted by the Chinese Representative Office.)
\end{abstract}

Key Words: Production Line, Scheduling Optimization, Bottleneck Identification, Improved Genetic Algorithm, Computer Simulation, Multivariate, Hierarchical Clustering

\section{INTRODUCTION}

In the manufacturing industry, the production scheduling is a resource allocation problem in which the various resources are assigned reasonably and orderly to different tasks in the workshop according to needs, such that the operation system can achieve the optimal state at completion and the best production efficiency with the minimal cost $[1,2]$. In the production system, the production efficiency and product quality directly hinge on the maximum output of the production line and the scheduling of the production process [3, 4]. The system performance, especially the output, is mainly dampened by limitations on production hardware and defects in production management $[5,6]$. If the production scheduling is unreasonable and its performance indices are not fully utilized, it is impossible for the production enterprise to achieve the optimal processing and manufacturing, not to mention the pursuit of higher profits $[7,8]$.

In general, the bottlenecks of production line mainly fall into three categories: equipment bottleneck (e.g. the machines with the lowest processing efficiency, the highest load, and the longest mean waiting time), output bottleneck (e.g. the machines with the highest blocking or starvation probability and the highest sensitivity) and time bottleneck [9-12]. In actual production, several kinds of bottlenecks may coexist in the production system. However, the existing recognition and prediction methods for production line bottlenecks can only identify 
one type of bottlenecks at a time. With the development of large-scale production lines, it is virtually impossible to interpret the actual production status with the existing bottleneck recognition theories [13-15].

Production line scheduling mainly includes single-machine scheduling, job scheduling, and parallel scheduling [16-19]. These scheduling strategies aim to tackle the numerous uncertainties in actual production, such as processing blocking, machine failure, uncertain procedure and other external disturbances [20-22]. One of the most effective scheduling approaches is the open production scheduling (OPS). Inspired by the traditional flexible jobshop scheduling problem (JSSP), the OPS is a novel scheduling method [23-28] that greatly enhances the processing efficiency of the entire system through the adoption of flexible processing route. Of course, the OPS raises a high demand for macro-optimization scheduling based on big data and features complex computation. Over the years, many intelligent optimization algorithms have been successfully applied to further improve the OPS's processing optimization ability, namely, competitive genetic algorithm (GA), directed ant colony algorithm (ACA), swarm intelligence (SI) information sharing algorithm, multiobjective simulated annealing algorithm [29-34]. Due to the high cost of field tests, computer simulation is the most common method for optimizing production line scheduling.

In light of the above, this paper attempts to overcome the defects of bottleneck recognition and scheduling optimization in open production line. For this purpose, the author analysed the impact mechanism of external disturbances and system configuration changes on the production line, and put forward a multi-bottleneck identification model for production line through computer simulation. Then, the proposed model was applied to optimize the scheduling of open production line. The research findings provide new insights into the efficient operation of production line.

\section{MULTI-BOTTLENECK IDENTIFICATION MODELLING AND SIMULATION FOR PRODUCTION LINE}

Fig. 1 presents the relationship between machine cluster (MC) and bottleneck cluster (BC).

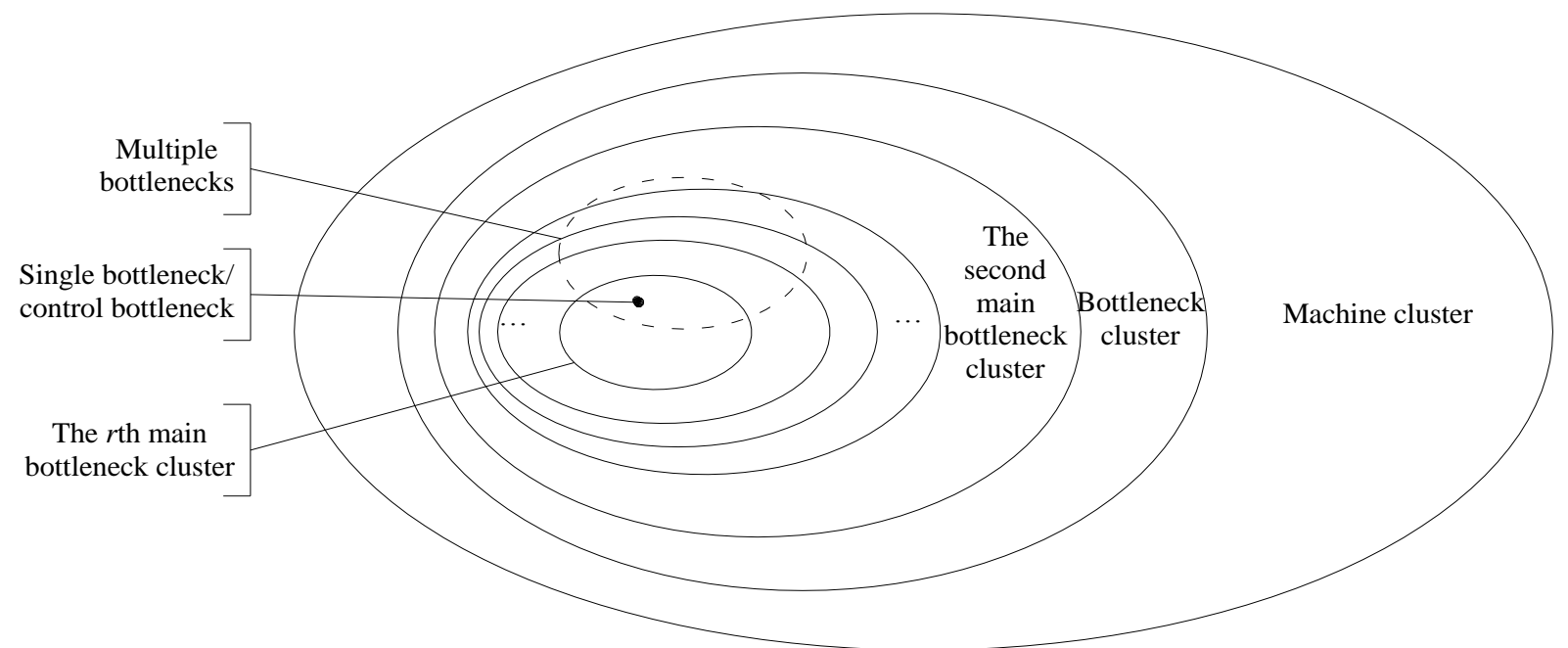

Figure 1: Relationship between MC and BC.

The MC refers to a collection of machines with similar functions on the production line. Let $\mathrm{C}=\left\{\mathrm{C}_{1}, \mathrm{C}_{2}, \ldots, \mathrm{C}_{k}, \ldots, \mathrm{C}_{n}\right\}$ be a set of a type of machines, with $G_{n}$ be the evaluated bottleneck value of $\mathrm{C}_{n}$. The following formula can determine whether an $\mathrm{MC}$ is a $\mathrm{BC}$ or non$\mathrm{BC}(\mathrm{NBC})$ : 


$$
\left\{\begin{array}{l}
B C: \quad G_{C_{k}}>\max \left\{G_{C_{u}}, u \in n, u \neq k, \forall u\right\} \\
N B C: \quad G_{C_{k}}>\max \left\{G_{C_{u}}, u \in n, u \neq k, \forall u\right\}
\end{array}\right.
$$

The machine with the largest $G_{n}$ in set $\mathrm{C}$ can be defined as the main bottleneck cluster.

Fig. 2 is the flow chart on the simulation of the proposed production line bottleneck identification model. It can be seen that the simulation process can be divided into the preprocessing phase, machine clustering phase and bottleneck recognition phase.

a) Pre-processing

The bottleneck identification of production line is the core premise of production scheduling. This section uses the logic of immune evolutionary algorithm to identify relevant attributes of machines on the production line, and builds a unified framework for machine clustering and bottleneck identification. Let $\mathrm{X}=\left\{\mathrm{X}_{1}, \mathrm{X}_{2}, \ldots, \mathrm{X}_{k}, \ldots, \mathrm{X}_{n}\right\}$ be the set of the relevant attributes of an $\mathrm{MC}$ on a certain production line. Then, the function of attribute matrix (FAM) can be expressed as:

$$
F A M=\left(x_{i j}\right)_{m \times n}
$$

The standardized form of Eq. (2) applies to the decision-making of machine attributes and bottleneck identification.

b) Machine clustering

After all the machines on the production line had been pre-processed, the machines with similar attributes were allocated into the same group by hierarchical clustering method. The distance between adjacent $\mathrm{MCs}\left(\mathrm{C}_{\mathrm{p}}\right.$ and $\left.\mathrm{C}_{\mathrm{q}}\right)$ after grouping can be calculated as:

$$
D_{s l}\left(C_{p}, C_{q}\right)=\min _{x, y}\left\{d(x, y) \mid x \in C_{p}, y \in C_{q}\right\}
$$

where $d(x, y)$ is the distance between two objects $\mathrm{x}$ and $\mathrm{y}$.

$$
d_{E}\left(A_{p}, A_{q}\right)=\sqrt{\sum_{j=1}^{n}\left(x_{p j}-x_{q j}\right)^{2}}
$$

The cophenetic correlation coefficient $(C P C C)$ was adopted to evaluate the granularity partitioning of MCs during hierarchical clustering.

$$
C P C C=\frac{(1 / M) \sum_{i=1}^{N-1} \sum_{j=i+1}^{N} P(i, j) P_{C}(i, j)-\mu_{P} \mu_{C}}{\sqrt{\left[(1 / M) \sum_{i=1}^{N-1} \sum_{j=i+1}^{N}[P(i, j)]^{2}-\mu_{P}^{2}\right]\left[(1 / M) \sum_{i=1}^{N-1} \sum_{j=i+1}^{N}\left[P_{C}(i, j)\right]^{2}-\mu_{C}^{2}\right]}},-1 \leq C P C C \leq 1
$$

where $P_{C}$ is the clustering matrix; $P(i, j)$ is the distance between vectors; $N$ is the number of data; $\mu_{c}$ and $\mu_{p}$ is the matrix mean values.

where $M=0.5 N(N-1)$.

$$
\left\{\begin{array}{l}
\mu_{P}=(1 / M) \sum_{i=1}^{N-1} \sum_{j=i+1}^{N} P(i, j) \\
\mu_{C}=(1 / M) \sum_{i=1}^{N-1} \sum_{j=i+1}^{N} P_{C}(i, j)
\end{array}\right.
$$

c) Bottleneck identification

The membership of each machine in a MC was calculated one by one. Through the calculation, the MCs were identified as $\mathrm{BC}$ or NBC. Then, all the MCs belonging to $\mathrm{BC}$ were ranked by importance and scale, and all the MCs belonging to NBC were ranked in the same manner. In this way, the main bottleneck(s) of the production line can be obtained.

Next, the proposed bottleneck identification method was validated with a 5-machine production line (Fig. 3). 


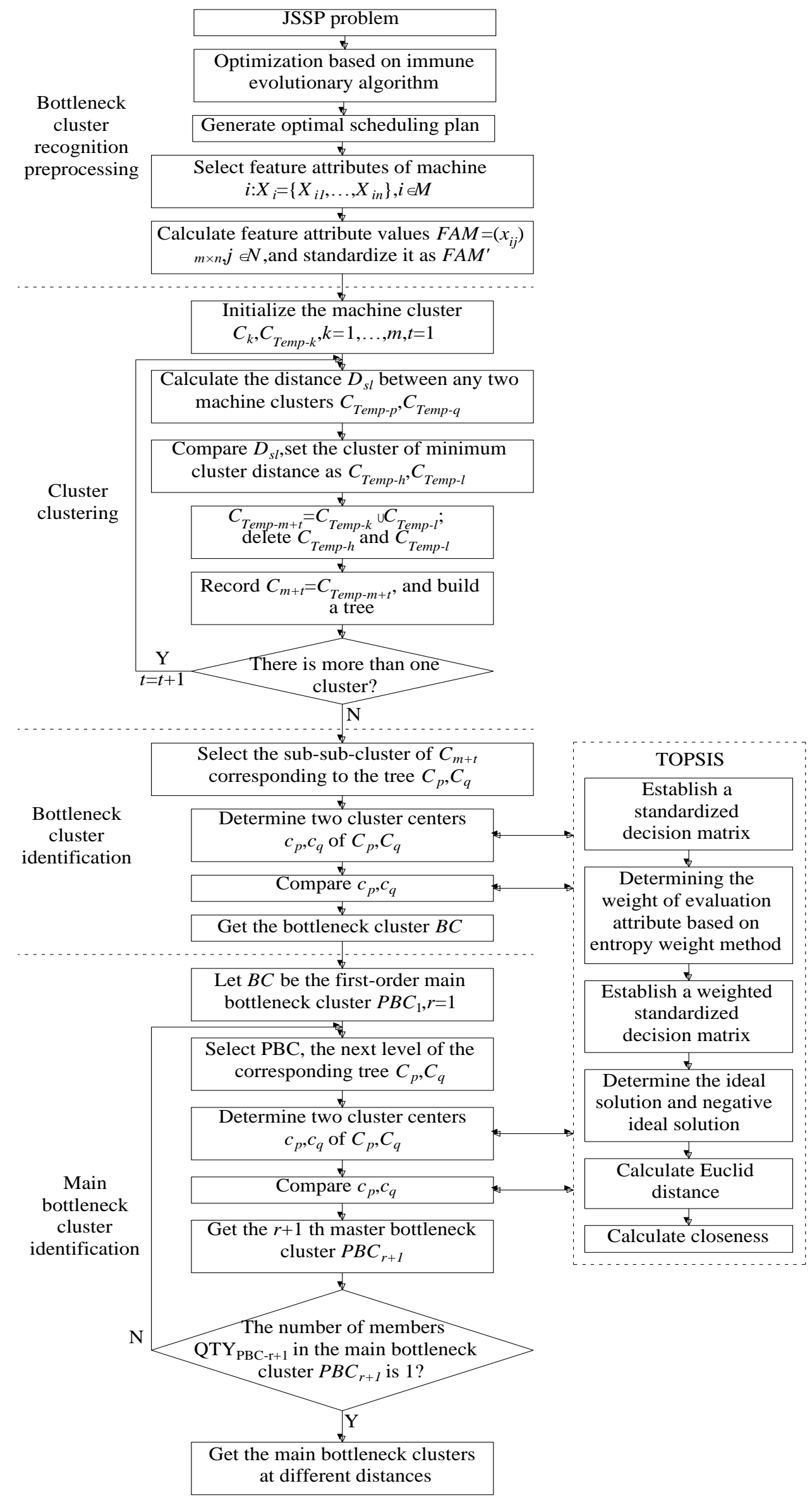

Figure 2: Flowchart of the simulation of production line bottleneck identification. 


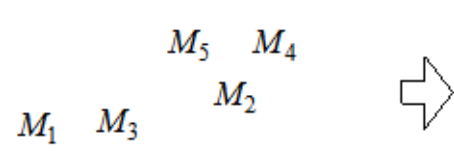

a The first stage Pretreatment

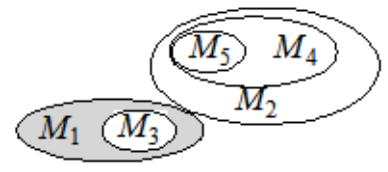

d The first stage Main bottleneck cluster identification

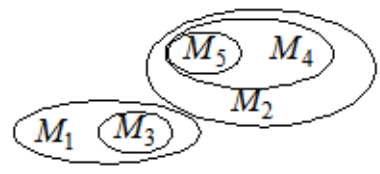

b The 2nd stage Cluster clustering

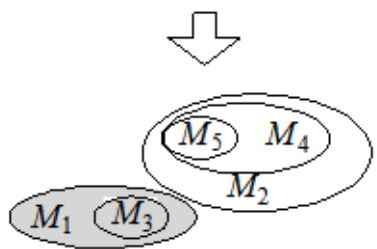

c The 3rd stage

Bottleneck cluster identification

Figure 3: Bottleneck identification process of a 5-machine production line.

Ten standard examples were selected from the JSSP for analysis, and the proposed algorithm was contrasted with two traditional algorithms (i.e. mobile bottleneck method and orthogonal test method). The comparison results are listed in Table I.

Fig. 4 presents the tree diagram of hierarchical clustering and recognized bottlenecks of production line in the $9^{\text {th }}$ example.

From Table I and Fig. 4, it can be seen that the proposed production line bottleneck identification algorithm has clear primary and secondary logics; the number of main bottleneck clusters decreased with the increase in the order, such that the primary bottleneck cluster was eventually recognized. Through multi-attribute decision-making, the proposed method can identify the overall attributes of the machines.

It can also be seen from the table that there is no direct connection between the machine load and bottleneck, and the $C P C C$ values obtained by our algorithm were all close to 1 . All these result prove the feasibility of our algorithm.

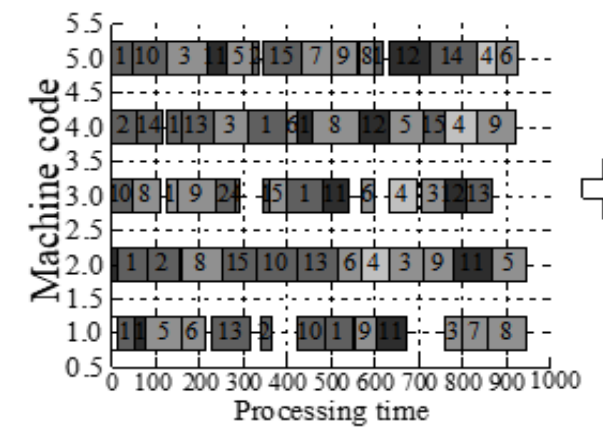

a Initialize based on LA 09's optimal scheduling results and the selected machine's attribute properties

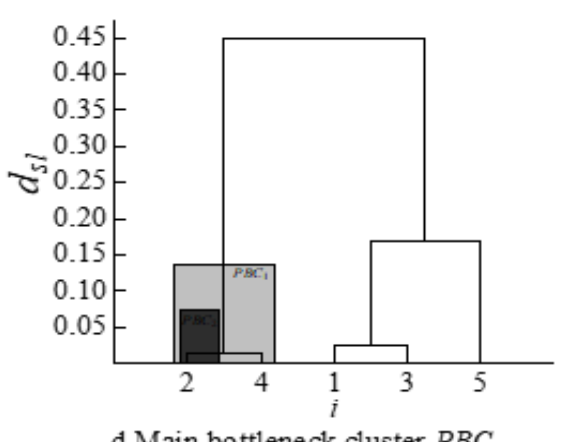

d Main bottleneck cluster $P B C_{2}$, distance $0.0261, r^{\prime}=2$

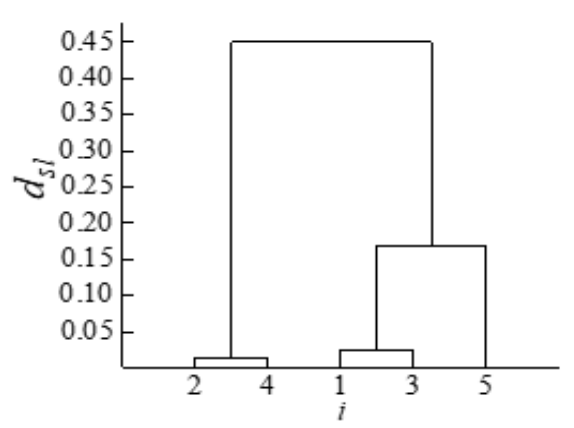

b LA09 level clustering results

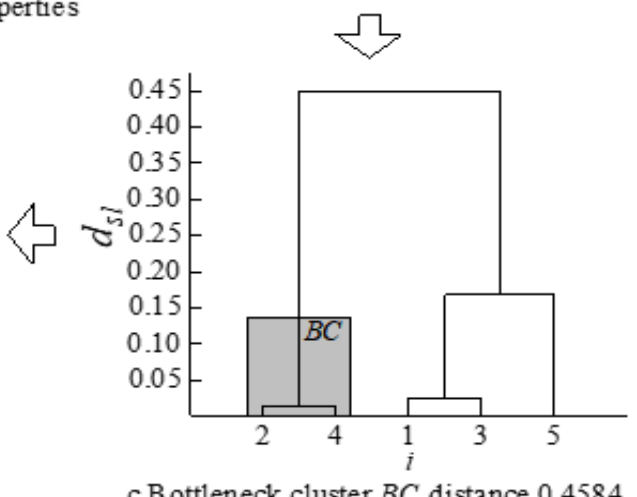

Figure 4: Tree diagram of hierarchical clustering and bottleneck identification simulation $\left(9^{\text {th }}\right.$ example). 
Table I: Comparison of bottleneck recognition results of three algorithms.

\begin{tabular}{|c|c|c|c|c|c|c|}
\hline \multirow[b]{2}{*}{ Examples } & \multirow[b]{2}{*}{$\begin{array}{l}\text { Scale } \\
(n \times m)\end{array}$} & \multirow[b]{2}{*}{$\begin{array}{l}\text { Bottlenecks identified } \\
\text { by the mobile } \\
\text { bottleneck method }\end{array}$} & \multirow{2}{*}{$\begin{array}{l}\text { Orthogonal test } \\
\text { method to } \\
\text { identify the } \\
\text { bottleneck }\end{array}$} & \multicolumn{3}{|c|}{ Compared methods } \\
\hline & & & & $\begin{array}{c}\text { Main } \\
\text { bottleneck } \\
\text { cluster }\end{array}$ & $\begin{array}{l}\text { Euclidean } \\
\text { distance }\end{array}$ & CPCC \\
\hline 1 & $10 \times 5$ & 6 & 6 & 6 & 0.5143 & 0.9971 \\
\hline 2 & $10 \times 5$ & 5 & 2 & 5 & 0.6745 & 0.9724 \\
\hline 3 & $10 \times 5$ & 4,2 & $6,2,4$ & 2 & 0.4326 & 0.9603 \\
\hline 4 & $10 \times 10$ & 4,2 & 2,4 & 4 & 0.4478 & 0.9765 \\
\hline 5 & $10 \times 10$ & 5 & 5 & 5 & 0.5683 & 0.9927 \\
\hline \multirow{2}{*}{6} & \multirow{2}{*}{$10 \times 10$} & \multirow{2}{*}{5} & \multirow{2}{*}{2} & 2,5 & 0.2899 & \multirow{2}{*}{0.9518} \\
\hline & & & & 2 & 0.0476 & \\
\hline 7 & $15 \times 5$ & 2 & 2 & 2 & 0.4988 & 0.9802 \\
\hline 8 & $15 \times 5$ & 2 & 2 & 2 & 0.6854 & 0.9743 \\
\hline \multirow{2}{*}{9} & \multirow{2}{*}{$15 \times 5$} & \multirow{2}{*}{3} & \multirow{2}{*}{3} & 3,5 & 0.4836 & \multirow{2}{*}{0.9948} \\
\hline & & & & 3 & 0.0147 & \\
\hline 10 & $15 \times 5$ & 6 & 4 & 5,6 & 0.3679 & 0.8947 \\
\hline
\end{tabular}

\section{SIMULATION OF MULTIVARIATE OPEN PRODUCTION LINE SCHEDULING OPTIMIZATION BASED ON IMPROVED GA}

\subsection{Construction of simulation model}

The open production line optimization can be expressed by the following mathematical model:

$$
\begin{gathered}
\text { Object } \min _{i \in J, j \in M}\left\{c_{i j}\right\} \\
c_{i k}-p_{i k}+L\left(1-x_{i h k}\right) \geq c_{i h}, \forall i \\
c_{l k}-c_{i k}+L\left(1-y_{i l k}\right) \geq p_{l h}, \forall k \\
c_{i j}, p_{i j}>0, i \in J, j \in M
\end{gathered}
$$

where $J$ and $M$ are the set of products and the set of machines, respectively. $L$ represents a sufficiently large positive number. Eq. (7) is the optimization objective function, it represents the minimization of the makespan; Eq. (8) represents that a product can only be processed by one machine at a certain time; while Eq. (9) represents that one machine can only process one product at the same time.

As shown in Fig. 5, the author established the multivariate open production line optimization based on improved GA. Specifically, the local optimum trap was avoided by enhancing population diversity; the iteration speed was accelerated with the introduction of adaptive crossover operator and genetic operator; without sacrificing the computing speed, the convergence quality was guaranteed through the addition of multivariate competition algorithm.

The specific steps of OPS optimization are as follows: (a) Initialize relevant parameters and population of the GA; (b) Decode the initial population based on the shortest queue priority logic to obtain the fitness $F(t)$ of each individual in the population; (c) Obtain a cross population $P_{c}(t)$ through adaptive crossover. The adaptive crossover operator can be expressed as:

$$
p c_{i, j}(t)=\left\{\begin{array}{lr}
K_{1} \sin \left(\frac{\pi}{2} \times \frac{f_{\max }(t)-f_{i, j}^{*}(t)}{f_{\max }(t)-f_{\text {avg }}(t)}\right)+\left(K_{2}-K_{1}\right), f_{i, j}^{*}(t)>f_{\text {avg }}(t) \\
K_{2}, \quad f_{i, j}^{*}(t) \leq f_{\text {avg }}(t)
\end{array}\right.
$$


(d) Find the fitness of $P_{c}(t)$ using the incremental decoding strategy; (e) Obtain a mutant population $P_{m}(t)$ through adaptive mutation, and compute its fitness through Step (d). The adaptive mutation operator can be expressed as:

$$
p m_{h}^{k}(t)=\alpha \times p m_{h}(t)+(1-\alpha) p m^{k}(t)
$$

(f) Combine the initial population with $P_{c}(t)$ and $P_{m}(t)$ into a new population WP; (g) Remove redundant individual from WP after the combination, increase population diversity by population diversity enhancement algorithm, and introduce multivariate competition operator to extract the optimal individuals after each iteration. The principles of multivariate competition operator are given in Fig. 6.

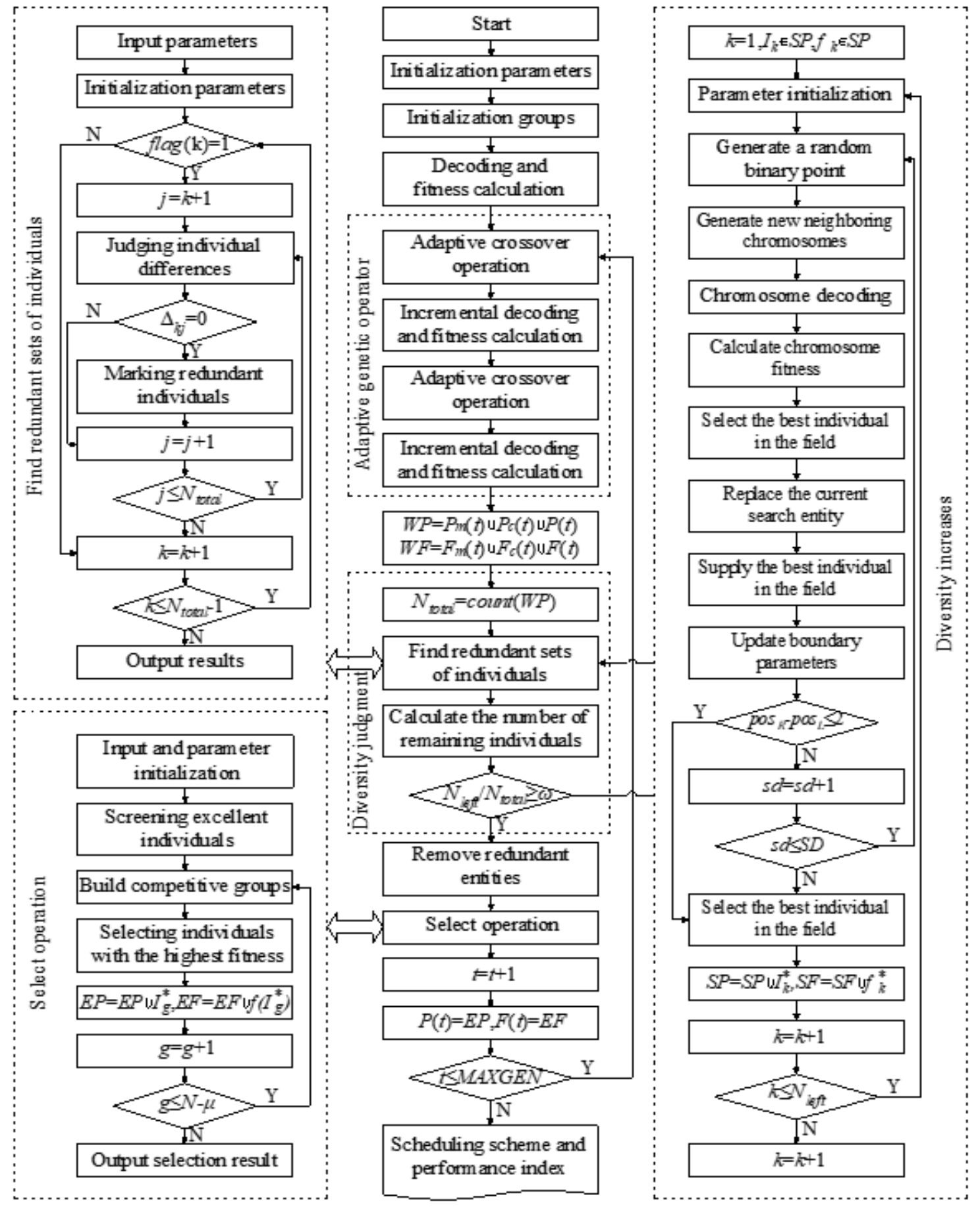

Figure 5: Flowchart of the simulation of multi-factor open production line optimization based on improved GA. 


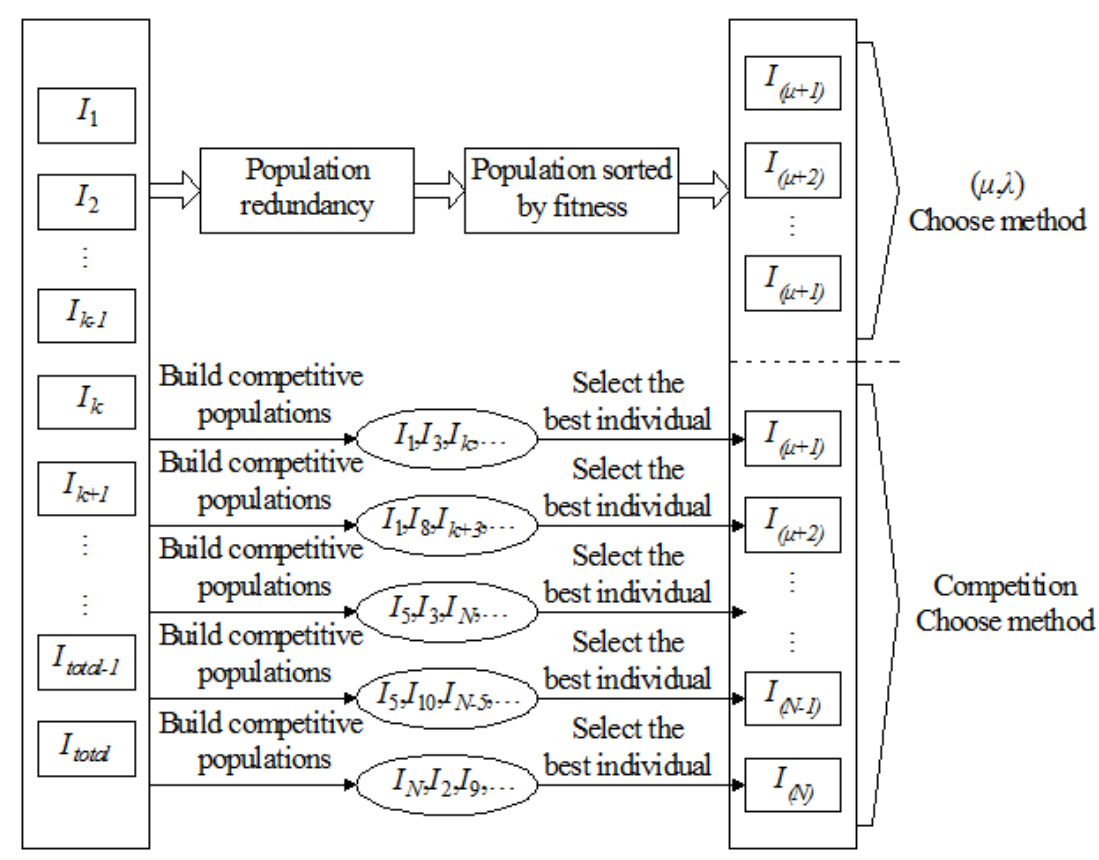

Figure 6: Principles of multivariate competition operator.

The population diversity can be evaluated with the independent individual density $\omega$ :

$$
\omega=N_{\text {left }} / N_{\text {total }}
$$

where $N_{\text {left }}$ and $N_{\text {total }}$ are the number of independent individuals and that of common individuals in the population, respectively. The value range of independent individual density is $0<\omega<1$.

The individual redundancy in the WP can be determined by the similarity factor:

$$
\Delta_{k j}=\left\|I_{k}-I_{j}\right\|_{1}=\sum_{i=1}^{m \times n}\left|g_{i}^{k}-g_{i}^{j}\right|
$$

Fig. 7 illustrates the principles of population diversity enhancement.

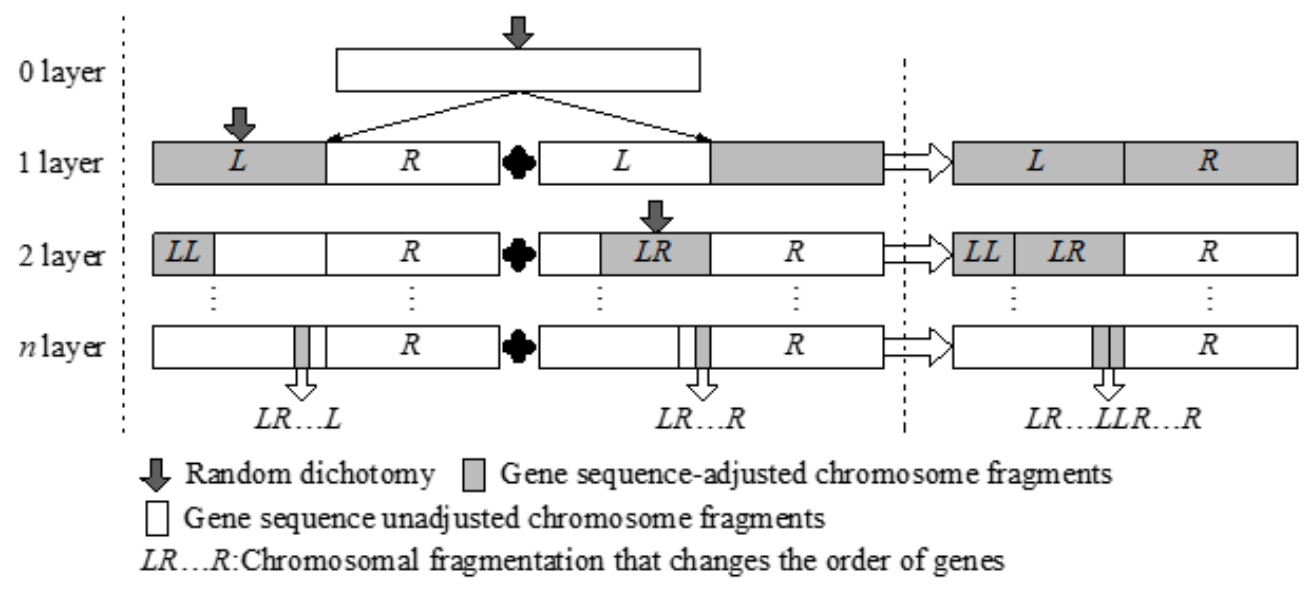

Figure 7: Principles of population diversity enhancement.

Repeat Steps (b)-(g) until reaching the pre-set threshold. The OPS optimization plan is thus obtained.

\subsection{Feasibility and effectiveness verification of the proposed simulation algorithm}

The proposed algorithm was compared with the GAs proposed by Liaw and Prins (GA-L and GA-P), ant colony optimization (ACO) and particle swarm optimization (PSO). The results 
are recorded in Table II. The theoretical best solution is denoted as BS. As shown in Table II, the proposed method can obtain the BS on any scale of the production line. This means the proposed method is stable and effective.

Table II: Comparison of the results of five algorithms.

\begin{tabular}{|c|c|c|c|c|c|c|}
\hline Size & BS & GA-L & GA-P & ACO & PSO & Proposed method \\
\hline $04 \times 04$ & 263 & 263 & 263 & 265 & 264 & 263 \\
\hline $05 \times 05$ & 315 & 316 & 317 & 315 & 315 & 315 \\
\hline $07 \times 07$ & 435 & 435 & 439 & 435 & 436 & 435 \\
\hline $10 \times 10$ & 602 & 602 & 604 & 602 & 602 & 602 \\
\hline $15 \times 15$ & 967 & 967 & 968 & 967 & 969 & 967 \\
\hline $20 \times 20$ & 1234 & 1236 & 1234 & 1234 & 1235 & 1234 \\
\hline Average gap/\% & & 0.018 & 0.389 & 0.102 & 0.023 & 0.000 \\
\hline
\end{tabular}

Next, the performance of the OPS optimization was further analysed from machine utilization rate $R_{M}$ and utilization variance $V_{M}$.

$$
\left\{\begin{array}{l}
\overline{R_{M}}=\frac{1}{m} \times \sum_{j=1}^{m} R_{M}(j) \\
R_{M}(j)=\sum_{i=1}^{m} p_{i, j} /\left(\max _{i}\left\{c_{i, j}\right\}-\min _{i}\left\{s_{i, j}\right\}\right) \\
V_{M}=\frac{1}{m} \times \sum_{j=1}^{m}\left(R_{M}(j)-\overline{R_{M}}\right)^{2}, j \in M
\end{array}\right.
$$

The machine utilization rate, service rate and their variances were calculated by Eqs. (15) and (16). The results (Figs. 8 and 9) show that, with the increase in the scale of the production line, the machine utilization rate gradually declined, while the product service rate first dropped and then increased. The variances of both indices were below 0.016 , and decreased with the expansion of the production line. The above trends reveal that the solution of the proposed method converges to the stable state, and verifies the correctness of the results in Fig. 8.

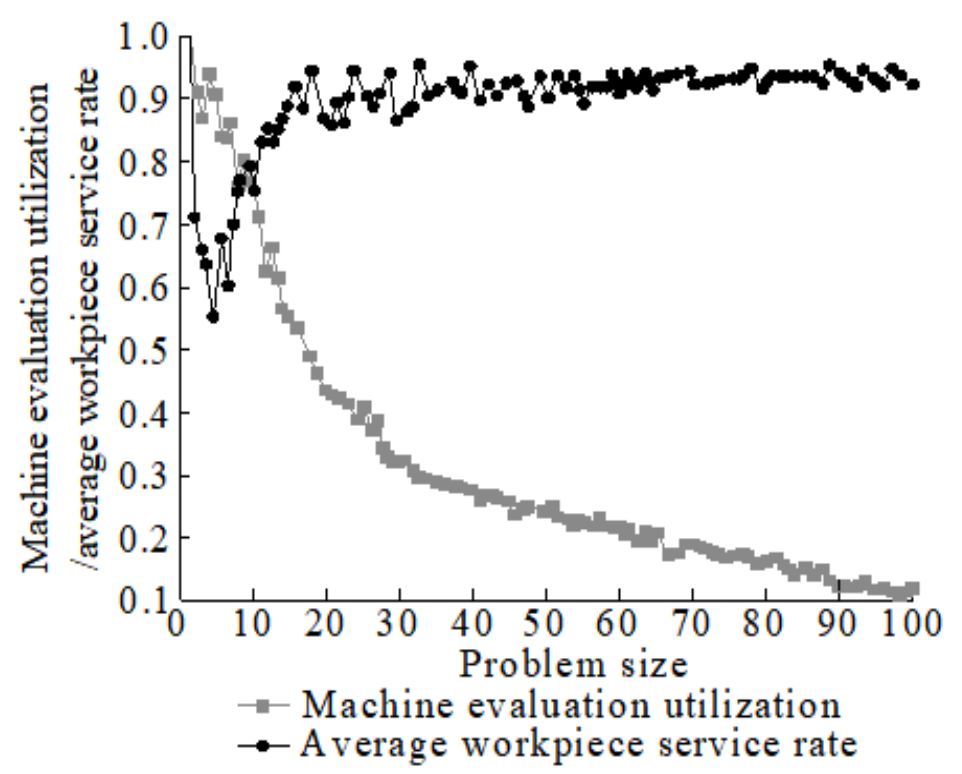

Figure 8: Variation in machine utilization rate and service rate with the scales of the production line. 


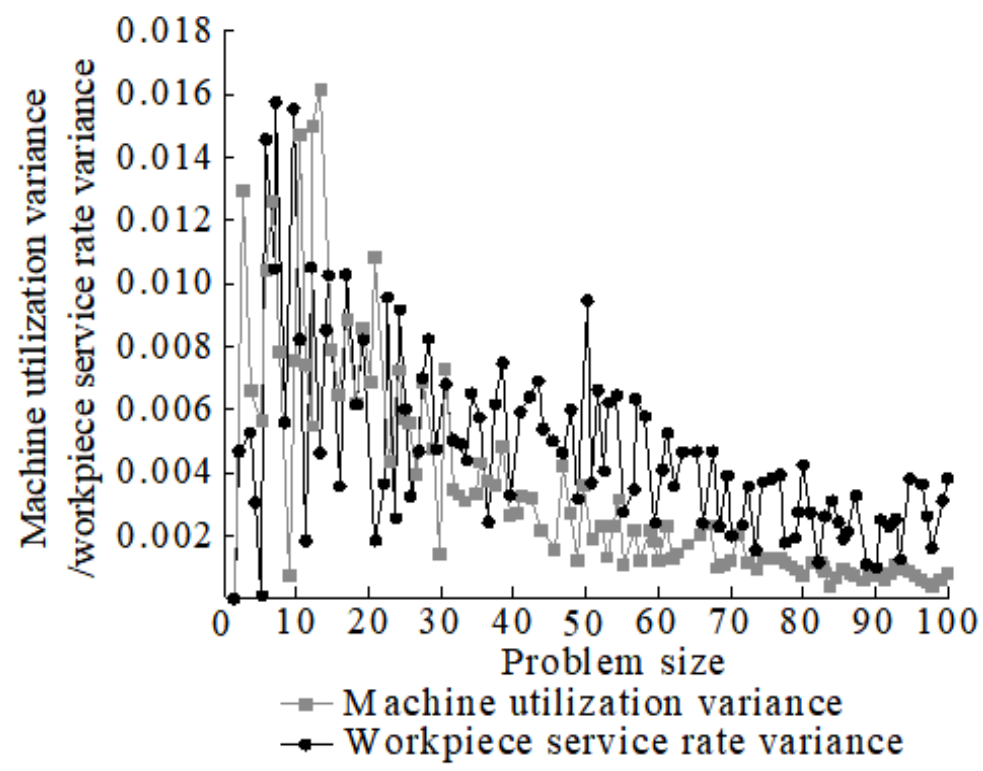

Figure 9: Variances of machine utilization rate and service rate at different scales of the production line.

\section{CONCLUSIONS}

Targeting the defects of bottleneck recognition and scheduling optimization in open production line, this paper analyses the impact mechanism of external disturbances and system configuration changes on the production line, and put forward a multi-bottleneck identification model for production line. Then, the proposed model was applied to optimize the scheduling of open production line. The purpose is to provide new insights into the efficient operation of production line. The main conclusions are as follows:

(1) The bottlenecks of production line were identified based on hierarchical clustering and multi-attribute decision-making, aiming to overcome the small candidate set and low accuracy of traditional bottleneck identification algorithms. The measured results show that the proposed algorithm has clear primary and secondary logics; the number of main bottleneck clusters decreased with the increase in the order; the number of machines in the main bottleneck cluster changed nonlinearly; there is no direct connection between the machine load and bottleneck; the $C P C C$ values were all close to 1 . All these results demonstrate the feasibility of the proposed algorithm;

(2) The traditional GA was improved in three aspects: the local optimum trap was avoided by enhancing population diversity; the iteration speed was accelerated with the introduction of adaptive crossover operator and genetic operator; without sacrificing the computing speed, the convergence quality was guaranteed through the addition of multivariate competition algorithm. Compared with the other four scheduling optimization algorithms, our method obtained the BS at any scale of the production line. The comparison proves the stability and effectiveness of the proposed algorithm.

\section{ACKNOWLEDGEMENTS}

The research is funded by Natural Science Fund of Hubei Province (No. 2017CFB598, No. 2017CFB677), the Natural Science Foundation of China (NSFC) (No. 61572012) and Science and Technology Research Project of the Education Department of Hubei Province (D20172203). 


\section{REFERENCES}

[1] Qassim, R. Y. (2000). The theory of constraints in manufacturing, Journal of the Brazilian Society of Mechanical Sciences, Vol. 22, No. 4, 503-511, doi:10.1590/S010073862000000400001

[2] Gerstl, E.; Mosheiov, G.; Sarig, A. (2014). Batch scheduling in a two-stage flexible flow shop problem, Foundations of Computing and Decision Sciences, Vol. 39, No. 1, 3-16, doi:10.2478/ fcds-2014-0001

[3] Kovács, G. (2017). Application of lean methods for improvement of manufacturing processes, Academic Journal of Manufacturing Engineering, Vol. 15, No. 2, 31-36

[4] Gong, D.; Tang, M.; Liu, S.; Li, Q. (2017). Reconsidering production coordination: A principalagent theory-based analysis, Advances in Production Engineering \& Management, Vol. 12, No. 1, 51-61, doi:10.14743/apem2017.1.239

[5] Gavareshki, M. H. K.; Zarandi, M. H. F. (2008). A heuristic approach for large scale job shop scheduling problems, Journal of Applied Sciences, Vol. 8, No. 6, 992-999, doi:10.3923/ jas.2008.992.999

[6] Mönch, L.; Schabacker, R.; Pabst, D.; Fowler, J. W. (2007). Genetic algorithm-based subproblem solution procedures for a modified shifting bottleneck heuristic for complex job shops, European Journal of Operational Research, Vol. 177, No. 3, 2100-2118, doi:10.1016/j.ejor.2005.12.020

[7] Zheng, Y. (2018). Enhancing innovation behaviour of enterprise personnel through neurofeedback training based on electroencephalography, NeuroQuantology, Vol. 16, No. 3, 6167, doi:10.14704/nq.2018.16.3.1191

[8] Du, Y., Chen, Q.-X., Mao, N. (2015). The research of variable production line into multi-channel, Academic Journal of Manufacturing Engineering, Vol. 13, No. 4, 6-12

[9] Wang, J.-Q.; Chen, J.; Zhang, Y.; Huang, G. Q. (2016). Schedule-based execution bottleneck identification in a job shop, Computers \& Industrial Engineering, Vol. 98, 308-322, doi:10.1016/j.cie.2016.05.039

[10] Dauzere-Peres, S.; Lasserre, J.-B. (1993). A modified shifting bottleneck procedure for job-shop scheduling, International Journal of Production Research, Vol. 31, No. 4, 923-932, doi: $10.1080 / 00207549308956766$

[11] Liu, M.-Z.; Tang, J.; Ge, M.-G.; Jiang, Z.-Q.; Hu, J.; Ling, L. (2009). Dynamic prediction method of production logistics bottleneck based on bottleneck index, Chinese Journal of Mechanical Engineering, Vol. 22, No. 5, 710-716, doi:10.3901/cjme.2009.05.710

[12] Mason, S. J.; Fowler, J. W.; Carlyle, W. M. (2002). A modified shifting bottleneck heuristic for minimizing total weighted tardiness in complex job shops, Journal of Scheduling, Vol. 5, No. 3, 247-262, doi: $10.1002 /$ jos.102

[13] Zuo, Y.; Gu, H.-Y.; Xi, Y.-G. (2006). Modified bottleneck-based procedure for large-scale flowshop scheduling problems with bottleneck, Chinese Journal of Mechanical Engineering, Vol. 19, No. 3, 356-361, doi:10.3901/cjme.2006.03.356

[14] Nahavandi, N.; Zegordi, S. H.; Abbasian, M. (2016). Solving the dynamic job shop scheduling problem using bottleneck and intelligent agents based on genetic algorithm, International Journal of Engineering - Transactions C: Aspects, Vol. 29, No. 3, 347-358, doi:10.5829/ idosi.ije.2016.29.03c.09

[15] Li, L. (2009). Bottleneck detection of complex manufacturing systems using a data-driven method, International Journal of Production Research, Vol. 47, No. 24, 6929-6940, doi: $10.1080 / 00207540802427894$

[16] Crăciunean, D. C.; Crăciunean, V. (2016). Membrane modelling for batch manufacturing scheduling, Academic Journal of Manufacturing Engineering, Vol. 14, No. 3, 36-45

[17] Shabtay, D.; Steiner, G.; Zhang, R. (2016). Optimal coordination of resource allocation, due date assignment and scheduling decisions, Omega, Vol. 65, 41-54, doi:10.1016/j.omega.2015.12.006

[18] Li, Y. G.; Zhang, M. S. (2017). A multi-objective lot-streaming optimization scheduling model considering the blocking effect, International Journal of Simulation Modelling, Vol. 16, No. 4, 731-741, doi:10.2507/IJSIMM16(4)CO19

[19] Cogliandro, S.; Cravero, C.; Marini, M.; Spoladore, A. (2017). Simulation strategies for regenerative chambers in glass production plants with strategic exhaust gas recirculation system, 
International Journal of Heat and Technology, Vol. 35, Special issue 1, S449-S455, doi:10.18280/ijht.35Sp0161

[20] Tang, M.; Gong, D.; Liu, S.; Zhang, H. (2016). Applying multi-phase particle swarm optimization to solve bulk cargo port scheduling problem, Advances in Production Engineering \& Management, Vol. 11, No. 4, 299-310, doi:10.14743/apem2016.4.228

[21] Khanzadi, M.; Soufipour, R.; Rostami, M. (2011). A new improved genetic algorithm approach and a competitive heuristic method for large-scale multiple resource-constrained projectscheduling problems, International Journal of Industrial Engineering Computations, Vol. 2, No. 4, 737-748, doi:10.5267/j.ijiec.2011.06.009

[22] Li, B. (2017). Energy saving optimization of machining center process route based on improved genetic algorithm, Academic Journal of Manufacturing Engineering, Vol. 15, No. 3, 35-42

[23] Singh, M. R.; Mahapatra, S. S.; Mishra, K. (2013). A novel swarm optimiser for flexible flow shop scheduling, International Journal of Swarm Intelligence, Vol. 1, No. 1, 51-69, doi:10.1504/ijsi.2013.055802

[24] Huang, S.; Tian, N.; Wang, Y.; Ji, Z. (2016). Multi-objective flexible job-shop scheduling problem using modified discrete particle swarm optimization, SpringerPlus, Vol. 5, No. 1, Paper 1432, 22 pages, doi:10.1186/s40064-016-3054-z

[25] Driss, I.; Mouss, K. N.; Laggoun, A. (2015). A new genetic algorithm for flexible job-shop scheduling problems, Journal of Mechanical Science and Technology, Vol. 29, No. 3, 1273-1281, doi:10.1007/s12206-015-0242-7

[26] Jiang, Z.; Zuo, L.; Mingcheng, E. (2014). Study on multi-objective flexible job-shop scheduling problem considering energy consumption, Journal of Industrial Engineering and Management, Vol. 7, No. 3, 589-604, doi:10.3926/jiem.1075

[27] Ahmadi, E.; Zandieh, M.; Farrokh, M.; Emami, S. M. (2016). A multi objective optimization approach for flexible job shop scheduling problem under random machine breakdown by evolutionary algorithms, Computers \& Operations Research, Vol. 73, 56-66, doi:10.1016/ j.cor.2016.03.009

[28] Tan, Y.; Mönch, L.; Fowler, J. W. (2018). A hybrid scheduling approach for a two-stage flexible flow shop with batch processing machines, Journal of Scheduling, Vol. 21, No. 2, 209-226, doi:10.1007/s10951-017-0530-4

[29] Mousavi, M.; Yap, H. J.; Musa, S. N.; Dawal, S. Z. M. (2017). A fuzzy hybrid GA-PSO algorithm for multi-objective AGV scheduling in FMS, International Journal of Simulation Modelling, Vol. 16, No. 1, 58-71, doi:10.2507/IJSIMM16(1)5.368

[30] Chauvet, E. (2018). Brain information optimization and ethical behavior, NeuroQuantology, Vol. 16, No. 3, 12-22, doi:10.14704/nq.2018.16.3.1158

[31] Liang, C. H.; Zeng, S.; Li, Z. X.; Yang, D. G.; Sherif, S. A. (2016). Optimal design of plate-fin heat sink under natural convection using a particle swarm optimization algorithm, International Journal of Heat and Technology, Vol. 34, No. 2, 275-280, doi:10.18280/ijht.340217

[32] Sen, G. D.; Sharma, J.; Goyal, G. R.; Singh, A. K. (2017). A multi-objective PSO (MOPSO) algorithm for optimal active power dispatch with pollution control, Mathematical Modelling of Engineering Problems, Vol. 4, No. 3, 113-119, doi:10.18280/mmep.040301

[33] Wang, T.-C., Xie, Y.-Z. (2016). BP-GA data fusion algorithm studies oriented to smart home, Mathematical Modelling of Engineering Problems, Vol. 3, No. 3, 135-140, doi:10.18280/ mmep.030304

[34] Zhang, X. H. (2018). Research on the evaluation and decision-making of product premium based on electroencephalography (EEG) testing technology, NeuroQuantology, Vol. 16, No. 3, 68-74, doi: $\underline{10.14704 / n q .2018 .16 .3 .1199}$ 